\title{
Students' reasoning when tackling electric field and potential in explanation of de resistive circuits
}

\author{
Ane Leniz, Kristina Zuza, and Jenaro Guisasola \\ Donostia Physics Education Research Group and Applied Physics Department, \\ School of Engineering Gipuzkoa (UPV/EHU), Plaza Europa 1, San Sebastian 20018, Spain
}

(Received 10 February 2017; published 19 May 2017)

\begin{abstract}
This study examines the causal reasoning that university students use to explain how dc circuits work. We analyze how students use the concepts of electric field and potential difference in their explanatory models of dc circuits, and what kinds of reasoning they use at the macroscopic and microscopic levels in their explanations. This knowledge is essential to help instructors design and implement new teaching approaches that encourage students to articulate the macroscopic and microscopic levels of description. A questionnaire with an emphasis on explanations was used to analyze students' reasoning. In this analysis of students' reasoning in the microscopic and macroscopic modeling processes in a dc circuit, we refer to epistemological studies of scientific explanations. We conclude that the student explanations fall into three main categories of reasoning. The vast majority of students employ an explanatory model based on simple or linear causality and on relational reasoning. Moreover, around a third of students use a relational reasoning that relates two magnitudes current and resistance or conductivity of the material, which is included in a macroscopic explanatory model based on Ohm's law and the conservation of the current. In addition, few students situate the explanations at the microscopic level (charges or electrons) with unidirectional cause-effect reasoning. This study looks at a number of aspects that have been little mentioned in previous research at the university level, about the reasoning types students use when establishing macro-micro relationships and some possible difficulties with complex reasoning.
\end{abstract}

DOI: 10.1103/PhysRevPhysEducRes.13.010128

\section{INTRODUCTION}

The explanation of simple dc electric circuits with resistors is a complex topic in physics, which involves physical laws, microscopic model of matter, and coherence in linking different concepts of electricity. When students reason about electric circuits, they have to integrate and apply basic concepts such as electric charge, electric potential, electric current, and electric field. Previous research on students' understanding of dc resistive circuits has been developed with primary and secondary students' models of current [1-4]. It is also consistently reported that many students at the secondary and university levels use electricity in a fragmented way, without linking electrostatics and electrodynamics. Students tend to regard chapters of electrostatics and dc circuits as two separate areas [5-9]. Therefore, clarifying students' understanding of electric circuits is one of the most important topics in physics education research [10].

Regarding previous studies, it is well established that electric potential is one of the concepts giving students the

Published by the American Physical Society under the terms of the Creative Commons Attribution 4.0 International license. Further distribution of this work must maintain attribution to the author(s) and the published article's title, journal citation, and DOI. greatest learning difficulties when interpreting electric circuits. Moreover, students are unable to define consistently current and potential differences, and they do not get to discriminate clearly between them. In addition, students frequently do not understand that the potential difference between two points in a circuit depends on its topology $[2,11,12]$. Härtel [13] found that students conceive the battery of a de circuit as a device that supplies "constant current" rather than one that constantly maintains the potential difference between its poles. Several studies on students' ideas about electrical circuits found that many students' explanations are usually based on electricity, as flow, describing macrolevel phenomena, but their explanations are not concerned with the microlevel nature of charges and the electric field acting on them [14].

Some studies point out that, often, there is a discontinuity in the presentation of the electrostatics and electric circuit topics in standard introductory physics courses $[14,15]$. In electrostatics, the attention is focused on electric charges, on the electric field produced by distributed charges, and on electric potential. Then, in the next chapter, the instruction is focused on batteries, resistors, current, and capacitors. However, the role played by the electric field on the movement of electrons in the wire or the relations between electric field and potential difference through the circuit are rarely dealt with in textbooks at the introductory level. Of course, the vast majority of textbooks at the university level 
explicitly define the relationships between field, potential difference, and electric current, but more emphasis is often placed on the communication of quantitative relations among concepts and laws than the role played by the concepts and laws in the electron model that explain macroscopic phenomena with microscopic particles. In textbooks, this macro-micro relationship is explained in a single step [16]. For example, the electric current is defined as $I=n q v_{d} A$, at the microscopic level. Next, in Sec. II, the Ohm's law for resistive circuits is defined as $I=\Delta V / R$, at the macroscopic level [17], followed by the mathematical relationship between equations $I=\Delta V / R$ and $\vec{E}=\rho \vec{J}$ [18]. However, this step in the explanation process is a major leap as the level of abstraction increases considerably from the visible world to the abstract and microscopic level [19]. This downplaying the aspects macro-micro in modeling electric circuits may in part be due to not taking into account all the characteristics of the electron model. Traditional approaches of teaching electric circuits may justify this lack of emphasis by stating that in many instances students can obtain a correct answer in numerical exercises without being able to distinguish between the macro-micro aspects of the concepts (for example, using the application of Kirchhoff's laws). Therefore, it might be argued that it is not worthwhile to emphasize the subtle difference between the microscopic and macroscopic aspects of the concepts involved in electron models.

While proposals for not addressing the distinction between macro and micro characteristics of the model have some merit, we feel the arguments for making such distinctions are stronger. First, it is common practice in the teaching of thermodynamics to make a distinction between state variables at the macroscopic level and their interpretation at the microscopic level. For example, the variable "temperature" of a gas. The instructor usually draws a distinction between its macroscopic definition at the phenomenological level and the statistical notion of temperature related to the average kinetic energy of particles at the microscopic level. This microscopic definition of temperature is necessary to understand the internal heat energy of a gas as the kinetic energy of a perfect gas. Hence, it is difficult to argue that it is too demanding to distinguish between the macroscopic and microscopic aspects of the concepts, including in the electron model. On the other hand, there is often a need to find an alternative to a purely macroscopic description. We are often left at the macroscopic scale, with explanations such as "this law, or this other law, tells us that things have to be this way." These types of explanations are insufficient to satisfy students, especially when alternative conceptions appear on the way of learning.

Moreover, there has been a growing consensus that students benefit from being exposed to microlevel phenomena that govern electricity and dc circuits [20,21]. Being inherently abstract, electricity provides an excellent foundation for teaching model building and concept linking between macroscopic-level and microscopic-level descriptions. These models, such as the gradient surface chargebased micromodel or dc circuit theory using a field model $[22,23]$ are based on epistemological and content analysis and/or students' difficulties and reasoning and they aim to promote students' articulation of the macroscopic and microscopic levels of description [24-26]. These studies consider that an explanation is at the macroscopic level when it is focused on the physical quantities, such as resistance, current, and potential difference from the overall perspective of the circuit. They mention the conventional current and the quantities at the level of operational definitions (for example, Ohms' law or the formula that relates potential and field). They also consider that the explanation is at the microscopic level when it is focused at the local level in terms of electrons, electron current, or charge density [27]. It would clearly be advantageous if students were already used to seeing circuits as being driven by an electric field that acts on electrons at the microscopic level (whether or not this results in a conventional current at the macroscopic level). Instead, after covering electrostatics, students are often confronted with a new situation (e.g., electrokinetic phenomena) that not only leads to new phenomena (charges of movement in a wire) but also challenges their view that an electric field is required for a current to flow in a wire.

Our study addresses students' types of reasoning in the scientific explanation of dc resistive circuits. In particular, we analyze the kinds of reasoning that students use at the macroscopic and microscopic levels in their explanations, when tackling electric and potential difference concepts in the explanation of dc circuits. This study is part of a broader research project whose aim is to design, implement, and assess a teaching-learning sequence on electrical circuits for foundation level courses on physics at university.

The study presented here adds to prior research on the examination of students' reasoning on explanations of dc circuits in introductory physics courses for science and engineering. Using an open-ended questionnaire, we show in detail the reasoning that students receiving traditional instruction at the university level follow on microscopic modeling of physical processes for dc resistive circuits. We are particularly interested in the causal models that students use relating electric fields and potential difference quantities and macroscopic-microscopic explanations. The overall aims of this study are the following:

(i) to acquire a vision of students' reasoning when they use the concepts of electrical field and potential difference when explaining how dc circuits work;

(ii) to analyze and interpret students' responses to the questionnaire within the framework of studies on scientific reasoning;

(iii) to identify specific cues that will help to understand students' difficulties in learning explanatory scientific models of dc electrical circuits. 
This paper is organized as follows. First the theoretical framework that underpins this work will be discussed in Sec. II. This is followed (Sec. III) by a description of the research methodology, which includes a description of the data collection, questionnaire, and analysis procedure. In Sec. IV, each of these questions is analyzed as well as its relationship with the objectives of the study. The questionnaire results are shown in Sec. V, where examples are presented of student responses according to the epistemological framework selected. In Sec. VI we consider to what extent the types of reasoning are appropriate in order to interpret the results and we summarize our conclusions and potential implications for teaching.

\section{THEORETICAL FRAMEWORK}

Scientific reasoning is characterized by the use of models and modeling. Models are used by scientists as representations of how systems are structured and behave [28]. The educational value of models and modeling is accepted as an essential part of science education by the international community [29]. This emphasis on student modeling of concepts has been shown to result in deeper understanding. However, when we refer to students' types of reasoning in scientific explanation, we have in mind more than just fostering the process of building models; we want to analyze students' different types of reasoning that support the model. The teaching of resistive electrical circuits is an example of using scientific reasoning based on models. So, it is important to pose the question about students' causal reasoning during the modeling of dc resistive circuits. Students often provide explanations that are incorrect from the standpoint of physics.

These explanations are interpreted, according to the epistemology of science and cognitive psychology, as a consequence of the narrow range of types of causal models with which most students are familiar [30,31]. For instance, students use simple causal reasoning ( $A$ affects $B$ ) when faced with constraint system models such as Ohm's law or the gradient surface charge model, which would require a more complex reasoning. Most students are only familiar with relatively simple types of causal models, but many concepts and theories in science depend on styles that are substantially more complex in ways that we will define shortly. We consider the frameworks of the epistemology of science and cognitive psychology as suitable for an analysis of students' explanation in the domain of dc circuits.

There are several theoretical frameworks that can be used to investigate students' causal reasoning [30-33]. These frameworks about scientific explanations are not mutually exclusive and may even complement each other. Although there is no single epistemological or cognitive theory about how scientists reason, some types of reasoning can be summarized that are widely accepted by the research community. In this article, we analyze the students' answers to the questionnaire according to four types of causal reasoning that are widely accepted in epistemological studies of scientific explanations.

One of the types of reasoning that is termed "simple causal reasoning," establishes a causal relationship between a certain cause $C$ and a certain effect $E[30,31]$. This is simple causality offering an elementary explanation of the phenomenon. For example, "the electrical current produces the electric field in the wire" or "the potential difference generates the electric field." A second type of causal reasoning is based on the principle that the cause of the change observed in a system is to be found outside the system and the integration of the various agents constitutes its explanatory power; this is referred to as "linear causal reasoning." Halbwachs [30] defines this type of causal reasoning as the tendency to juxtapose a number of simple causal relations to form a new ordered relation, a causal chain in which every cause is the result of another cause, or every result is the cause of another result. Often, after establishing a few simple causal links $(A, B, C)$, the reasoning operates in a causal chain $A \sim B \sim C$ with one process producing the other either successively or simultaneously. For example, "the charge density distribution in the wire generates an electric field in the wire. This electric field produces the potential difference in each part of the circuit, depending on the resistance." A chain of simple causal links is constructed between the event $A$, the charge density distribution produces electric field and event $\mathrm{B}$, the electric field produces potential difference. This happens in each part of the circuit and so this linear reasoning has the form of a causal chain: charge density distribution electric field $\sim$ potential difference.

A third type of reasoning, "relational reasoning or relational causality", is used where the outcome is due to the relationship between two variables (physical quantities) of the system. Grotzer [31] finds that these relationships are not an explanation by themselves but have an explanatory character due to the prediction of how the system will behave. For example, "the electric field is the result of the relationship between the concentration of electrons and the width of the wire." In this explanation, the relationship between two variables (concentration of electrons and width of the wire) predicts the value of the electric field. In the case of relational reasoning, one needs the relationship between two variables of the system that leads to a result. Conversely, in the simple causal or linear causal reasoning, the principal characteristic is the link between two or more events.

Finally, in scientific inquiry we frequently find a fourth type of reasoning that Perkins and Grotzer [32] call "multilevel reasoning," where the description of the system is more refined, using different models of description that are interrelated; for example, Ohm's model, or the gradient surface charges micromodel as a framework for explaining the electric circuit processes. Thus, they correlate two different descriptions of the same system at two different 
levels of analysis (focuses on electron current at the local level, or on conventional current at the global systemic level). In this case the explanatory strength lies in the fact that the laws of the "deeper" model allow of the laws of the initial model to be deduced.

\section{THE STUDY}

To address the objectives of the study, we gave 141 students at the University of the Basque Country (Spain) a questionnaire (see Appendix) on dc resistive circuits as part of their first-year final exam. They had taken two years of physics at high school and received $3.5 \mathrm{~h}$ of lectures on electromagnetism and spent $2 \mathrm{~h}$ in the laboratory per week for 14 weeks in their second semester. Experienced teachers from the Department of Physics gave the lectures. The program electric current and direct current circuits were taught for two weeks of this course. The students used the textbook "Physics for scientists and engineers" by Tipler and Mosca [17]. The lectures, which include problem-solving exercises, dealt with current and the motion of charges, resistance, batteries, and Ohm's Law, combinations of resistors, Kirchhoff's rules, and $R C$ circuits. Traditional teaching presented the current as the movement of electrons through the wire and defines current at the microscopic level. In the following, Ohm's law is defined for resistive circuits at the macroscopic level and the equation $J=\sigma E$ at the microscopic level. The traditional teaching in the Physics Department also analyzed how to use Kirchhoff's rules to calculate the energy balance in the circuit at the macroscopic level, and examples similar to those appearing in textbooks were given [17]. Situations where it is useful to take into account energy supplied by the battery and the energy consumed by resistors were presented and analyzed at the macroscopic level and with experimental data.

Once the questionnaire had been prepared, it was validated in terms of contents and aims. First, experienced members of the Applied Physics Department answered the questionnaire and made suggestions that were considered in the final draft of the questionnaire. Second, we carried out a dry run with first-year course students, which confirmed that students had no problem understanding how the questions were formulated. Finally, these questions were included in the first-year students' end of term examination.

In order to characterize the responses, the comments recognized as "an explanation" were coded, based on categories with easily recognizable features, such as scientific statements and argumentation from the four types of reasoning defined in the theoretical framework [30,31]. This involved one member of the research team reading the students' answers and deriving a draft set of description categories for each question. The same researcher then reread the students' answers and tentatively allocated each answer to one of the draft categories. The other researchers carried out the latter task independently. Once the answers had been classified, answer allocations were compared and a very significant degree of agreement was reached with a Cohen's kappa reliability coefficient average of 0.86 . Any disagreements about category description or answer allocations were resolved by referring to the answers as the only evidence of students' understanding. The focus was on the students' understanding, taking the students' answer as a whole, rather than on the occurrence of particular statements corresponding to a specific category of explanation [34]. An iterative process was used to produce the finalcategory descriptions that reflected similar understanding among answers allocated to each category and the differences between the categories [35].

\section{EXPERIMENTAL DESIGN}

In this section, we describe the three questions completed by the students (see Appendix). Question Q1 sets out to explore the types of reasoning that students have in relating the concept of field that they have worked on in electrostatics with the same concept used in dc circuits. The aim of this question is to inquire if students are able to distinguish between the role of electric field in a cylindrical conductor in electrostatics and the role into the wire of a circuit in stationary state.

Questions Q2 and Q3 set out to explore the difficulties students have in relating the movement of charges in a dc electric circuit with the electric field that is generated inside the conductor wire, and with the potential difference between the two points of the circuit. The students are presented with two simple de circuits; one of them, in question Q2, with a carbon resistance, and the other, in question Q3, with a narrowing of the conductor wire (which acts as "resistance"). Question Q2 presents a situation of direct current in a static state in a simple dc circuit, which is familiar for the students in an academic context. Question Q3 presents a similar situation to that in Q2, but in this case the resistance is represented by a cable that is narrower than in the rest of the circuit. In both questions, the circuit has been connected for a long period, and therefore the current circulating through it is constant. In the first section, Q2A and Q3A, the students need to identify the diagram that correctly represents the electric field at two points of the circuit. In the second section, Q2B and $\mathrm{Q} 3 \mathrm{~B}$, they are asked about the meaning of the potential difference between two points of a circuit (Q3B) and, in particular, about the relation between the potential difference and the distribution of the charges in the cable of the circuit $(\mathrm{Q} 2 \mathrm{~B})$.

The students, in order to respond correctly to Questions Q2A and Q2B, can use a macroscopic or microscopic explanatory level. At the macroscopic level, the students must indicate that in the steady state the current is the same throughout the circuit and therefore the electric field in the resistance must be greater than in the copper cable so that 
the speed of the sea of electrons will be constant throughout the circuit. From a microscopic perspective, the students must bear in mind that the flow of electrons must be equal throughout the circuit: $n A u_{\mathrm{Cu}} E_{A}=n A u_{\mathrm{C}} E_{B}$. In question Q2A, carbon possesses much less mobility of the electrons $u_{\mathrm{C}}$ than copper $u_{\mathrm{Cu}}\left(u_{\mathrm{Cu}}>u_{\mathrm{C}}\right)$ and since the density of mobile electrons $n$ and the area $A$ of the cable section are equal, the electric field tends towards a uniform large magnitude throughout the carbon and uniform small magnitude throughout the copper $E_{A}<E_{B}$. In question Q3A, the cable section varies $A_{A}>A_{B}$ and so $E_{A}<E_{B}$. Both explanatory models have been studied by the students during the course and are the ones the students are expected to use to solve the problem in the question. However, as we shall see from the results, explanations of this type are practically nonexistent in their responses.

The analysis of the question can be made in greater depth if one takes into account the gradient surface charges model (see, for example, Chabay and Sherwood [26], Chap. 19, or Härtel [36]). However, we do not expect this type of explanation, as the students in the sample have not been exposed to this model.

In questions Q2B and Q3B, in order to answer correctly, students can explain at the macroscopic level that for the circulation of charges between two points of a conductor, there needs to be a potential difference between the two points. They can also establish a relation between the potential difference generated by the battery and the potential difference in the different parts of the circuit and the current. At the microscopic level, students can relate the variation of concentration of charges at the local level and the potential difference. They can remember from electrostatics that different distributions of charges in the space produce an electric field and so, a potential difference. In question Q2B, the students are explicitly asked to relate the electrical potential with the distribution of charges at the microlevel, while in question Q3B the question is more open ended and they can establish a relation at the macro- or microlevel.

\section{RESULTS AND DISCUSSION}

In this section, we give the results obtained for all three questions. As we commented before, the aim of the analysis is not to see how many students answered correctly and how many did not. It is a research focused on the analysis of students' reasoning to explain the behaviour of dc circuits. Thus, students' responses have been grouped into different types of reasoning linked with categories of explanations. We identify some ways of reasoning and conceptual difficulties, which seem to be common in many students. This description of students' ideas will concentrate on some persistent specific categories of ideas and how we might interpret them through the lens of four types of reasoning. It is important to notice that the argumentation of the explanation (i.e., the explanatory category) is directly related to the form of reasoning in the context of solving the question.

The results of the students' answers to the first questions are given as percentages in Table I.

Explanations in the first category (I) use a simple causal reasoning: "If there is current, there is an electric field." Examples of this type of response are

"An electric field appears in the cable because we have an electric current. In the electrostatic state the situation was stationary".

TABLE I. Types of reasoning and explanations in question Q1, which asks about the concept of electric field in electrostatics and dc circuit contexts.

\begin{tabular}{|c|c|c|}
\hline Type of reasoning & Category of explanation & Percentage $(N=141)$ \\
\hline $\begin{array}{l}\text { 1.1. Microlevel simple causal (gradient of } \\
\text { concentration of charges-electric field or } \\
\text { potential difference) }\end{array}$ & & 0.0 \\
\hline $\begin{array}{l}\text { 1.2. Macrolevel simple causal (current-electric } \\
\text { field) }\end{array}$ & $\begin{array}{l}\text { I. If there is a current, there is an } \\
\text { electric field }\end{array}$ & 39.0 \\
\hline $\begin{array}{l}\text { 2.1. Microlevel linear causal (gradient of } \\
\text { concentration of charges-potential difference) }\end{array}$ & & 0.0 \\
\hline $\begin{array}{l}\text { 2.2. Macrolevel linear causal (battery-potential } \\
\text { difference-electric field) }\end{array}$ & $\begin{array}{l}\text { II. The potential difference generates } \\
\text { the electric field in the cable. }\end{array}$ & 21.0 \\
\hline $\begin{array}{l}\text { 3.1. Microlevel relational causal (relation } \\
\text { between current and resistance produces field } \\
\text { or potential difference) }\end{array}$ & & 0.0 \\
\hline $\begin{array}{l}\text { 3.2. Macrolevel relational causal (relation } \\
\text { between current and resistance produces field } \\
\text { or potential difference) }\end{array}$ & & 0.0 \\
\hline No logical line of reasoning & & 17.0 \\
\hline No answer & & 23.0 \\
\hline
\end{tabular}


"The current in the wire produces an electric field. This situation is different from electrostatic situation, where the electric field was zero because there is not current".

In these explanations, a simple causal reasoning is established between a cause, "electric current" and an effect, "electric field". In the category of explanations I, the cause is a description of the physical entities presented in the question (phenomenological orientation), in this case the electric current, but the relation with the effect is not linked to relevant concepts or with other theoretical connections. These explanations focus at the macroscopic level; they fail to mention explicitly the mechanism that underlies the current producing the electric field.

Explanation II "the potential difference generates the electric field," states that the cause of the existence of the electric field in the cable is the potential difference generated by the battery. The justifications follow a linear causal reasoning: the battery generates potential difference and this in turn generates the electric field in the cable. The students' explanations are incorrect as the battery is not the only factor that is responsible for the electric field in the conductor wire. However, this is the explanation that is closest to the scientific one among the students' explanations. No explanation explicitly mentioned the underlying mechanism of the causal process. An example of this type of response is
"When there is a current flowing, it means that the cable is connected to a battery that generates a potential difference. The potential difference produces the electric field inside the cable, which makes the electrons move."

Just three responses establish mathematical relations between the electric field and electric potential. As an example,

"As the question states, the electric field $E$ is zero in the cylinder when it is in an electrostatic situation. Nevertheless, if current flows then it is no longer in equilibrium since there are electrons in movement. Also, if the charges move there is a potential difference between the opposite ends of the cylinder. Then: $V_{a b}=\int \vec{E} \cdot \overrightarrow{d l}$. If there is a potential difference. the field is other than zero"

The students' answers to questions Q2A and Q3A are shown in Table II.

Explanation I is very much a minority response and includes those responses that explain the value of the electric field based on the concentration of electrons inside the wire. This explanation includes a simple causal line of reasoning that postulates the concentration of electrons in the wire as the cause of the electric field: charge density distribution $\rightarrow$ electric field. This model focuses

TABLE II. Explanation categories to the relations between current and electric field in the first part of questions Q2A and Q3A.

\begin{tabular}{|c|c|c|c|}
\hline Type of reasoning & Category of explanation & $\begin{array}{l}\text { Q2A percentage } \\
\quad(N=141)\end{array}$ & $\begin{array}{l}\text { Q3A percentage } \\
\qquad(N=141)\end{array}$ \\
\hline $\begin{array}{l}\text { 1.1. Microlevel simple causal (gradient } \\
\text { of concentration of charges-electric } \\
\text { field or potential difference) }\end{array}$ & $\begin{array}{l}\text { I. The concentration or difference of } \\
\text { concentration of electrons in each part } \\
\text { of the circuit produces the electric field }\end{array}$ & 1.0 & 4.5 \\
\hline $\begin{array}{l}\text { 1.2. Macrolevel simple causal } \\
\text { (current-electric field) }\end{array}$ & II. The electric current generates electric field & 10.5 & 33.0 \\
\hline $\begin{array}{l}\text { 2.1. Microlevel linear causal } \\
\text { (gradient of concentration of } \\
\text { charges-potential difference) }\end{array}$ & & 0.0 & 0.0 \\
\hline $\begin{array}{l}\text { 2.2. Macrolevel linear causal } \\
\text { (battery-potential difference-electric } \\
\text { field) }\end{array}$ & & 0.0 & 0.0 \\
\hline $\begin{array}{l}\text { 3.1. Microlevel relational causal (relation } \\
\text { between current and resistance } \\
\text { produces field or potential difference) }\end{array}$ & & 0.0 & 0.0 \\
\hline \multirow{2}{*}{$\begin{array}{l}\text { 3.2. Macrolevel relational causal } \\
\text { (relation between current and resistance } \\
\text { produces field or potential difference) }\end{array}$} & $\begin{array}{l}\text { III.a. The electric field is proportional } \\
\text { to the conductivity of the material }\end{array}$ & 38.0 & \\
\hline & $\begin{array}{l}\text { III.b. The electric field value depends on the } \\
\text { width of the cable and the current }\end{array}$ & & 38.5 \\
\hline No logical line of reasoning & & 50.5 & 24.0 \\
\hline No answer & & 0.0 & 0.0 \\
\hline
\end{tabular}


its explanation at the microscopic level-on the concentration of electrons in the wire. The students make an erroneous interpretation that the distribution of the charges responsible for the electric field is to be found inside the wire. It should be stressed that these responses do not explain what the mechanism is that generates the distribution of the charge throughout the circuit and this leads to responses of all kinds. That is to say, with the same reasoning based on the misunderstanding of the concepts, they choose different options for the question. Most of the responses are like the examples shown below:

"Ea is less than Eb because the charge at A produces a larger electric field at $B$ because it is a larger charge. There is a greater concentration of charges in the carbon than in the conductor cable."

"A smaller charge flowing in the thick wire (B point) means there will be less electric field."

"The same charge flows through point $A$ and point $B$ therefore the electric field is the same at both points."

Category of explanation II brings together those responses that relate the value of the electric field with that of the current. The explanations in this category focus on establishing a simple causal reasoning (current $\rightarrow$ field) with a phenomenological orientation. This explanation is similar to the one we find in explanation I of question Q1. The explanations do not explicitly identify the underlying mechanism of the current that produces the electric field, but the vast majority $(85 \%)$ choose the option $E_{A}=E_{B}$. This example is typical of this category:

"The current in the circuit is constant, which means that the electric field must be equal at the two points A and B."

This type or reasoning pursues an overgeneralization of the empirical rule that states that in a steady state the amperage of the current is the same, then generalizes by stating that the magnitude of the electric field is likewise the same.

Explanation category III includes responses that set up a relation between the electric field and the conductivity of the material for question Q2A (explanation IIIa). For question Q3A, due to the analogy used, a similar type of reasoning is applied to the variable "cable width" (area of the cable section) (explanation IIIb). In both cases a relational reasoning is used to explain the response in terms of the relation between two variables: either conductivity or width and current, to arrive at a conclusion about the magnitude of the electric field [37]. However, the underlying mechanism of the functional relation is not described. This lack of justification of the relational mechanism leads the relational line of reasoning to choose different options $\left(56 \% E_{A}>E_{B}, 29 \%\right.$ $E_{A}<E_{B}, 15 \% E_{A}=E_{B}$ in $\mathrm{Q} 2 \mathrm{~A} ; 62 \% E_{A}>E_{B}, 13 \%$
$E_{A}<E_{B}, 25 \% E_{A}=E_{B}$ in Q3A). Examples of this type of response are

"The conductivity of carbon is less than that of copper. So the carbon needs a larger electric field than copper to maintain the same current." (question 2A)

"The E field in the smaller portion of the wire will be smaller because there is a smaller charge (current) in that portion." (question 3A)

" $E_{A}$ is less than $E_{B}$ because the electrons will accumulate progressively in the narrow section, thus creating a larger electric field." (question 3A)

The students' answers to questions Q2B and Q3B are shown in Table III.

Although question Q3B does not explicitly ask for a macro-micro relation in the answer, we have observed that the percentage of responses at the microscopic level is similar both in question Q3B and in Q2B. 12\% of explanations are in category $\mathrm{I}$, which consists in a simple causal reasoning that claims that the difference in the electron density is the cause of the potential difference: charge density distribution $\rightarrow$ the potential difference. In this kind of answer there is a single cause (concentration or concentration difference of charges) that produces a result (potential difference). For example,

"A bigger change in charge density means a larger potential difference." (question 2B)

"Yes, there is a potential difference because the charge density changes from point A to point $B$. The cable gets narrower." (question 3B)

Around $8 \%$ of the explanations are in explanation category II, which exhibits a linear causal reasoning in which a sequence is established: fromcharge density distribution $\rightarrow$ electric field $\rightarrow$ the potentialdifference. In these responses, a chain of simple causal links is constructed between the event "charge density distribution," the event "existence of electric field" and the event "generation of potential difference." For example,

"Yes, the charge density can affect the electric field and the potential difference depends on the electric field." (question 2B)

Explanation category III (around 25\%) is based on a relational reasoning, where the cause is the relation established by Ohm's law between the magnitudes current and resistance to affect the potential difference. For example,

"The amperage does not change in the circuit but between points $A$ and $B$ the resistance (width) changes, therefore, the potential difference also changes according to Ohm's law." (Question 3B) 
TABLE III. Categories of explanation to the definition of potential difference in Q3B and its relation to charges distributions in Q2B.

\begin{tabular}{|c|c|c|c|}
\hline Type of reasoning & Category of explanation & $\begin{array}{l}\text { Q2B percentage } \\
\quad(N=141)\end{array}$ & $\begin{array}{l}\text { Q3B percentage } \\
\quad(N=141)\end{array}$ \\
\hline $\begin{array}{l}\text { 1.1. Microlevel simple causal (gradient of } \\
\text { concentration of charges-electric field or } \\
\text { potential difference) }\end{array}$ & $\begin{array}{l}\text { I. The distribution of the charges } \\
\text { generates potential difference }\end{array}$ & 12.0 & 12.0 \\
\hline $\begin{array}{l}\text { 1.2. Macrolevel simple causal } \\
\text { (current-electric field) }\end{array}$ & & 0.0 & 0.0 \\
\hline $\begin{array}{l}\text { 2.1. Microlevel linear causal (gradient of } \\
\text { concentration of charges-potential } \\
\text { difference) }\end{array}$ & $\begin{array}{l}\text { II. Charge density distribution implies } \\
\text { electric field that generates the } \\
\text { potential difference }\end{array}$ & 8.0 & 7.0 \\
\hline $\begin{array}{l}\text { 2.2. Macrolevel linear causal } \\
\text { (battery-potential difference-electric field) }\end{array}$ & & 0.0 & 0.0 \\
\hline $\begin{array}{l}\text { 3.1. Microlevel relational causal (relation } \\
\text { between current and resistance produces } \\
\text { field or potential difference) }\end{array}$ & & 0.0 & 0.0 \\
\hline $\begin{array}{l}\text { 3.2. Macrolevel relational causal (relation } \\
\text { between current and resistance produces } \\
\text { field or potential difference) }\end{array}$ & $\begin{array}{l}\text { III. The potential difference between } \\
\text { two points of the circuit is measured by } \\
\text { Ohm's law (There is no relation } \\
\text { between the potential difference and } \\
\text { the concentration of charges in the } \\
\text { cable) }\end{array}$ & 25.0 & 27.0 \\
\hline No logical line of reasoning & & 31.0 & 27.5 \\
\hline No answer & & 24.0 & 26.5 \\
\hline
\end{tabular}

In explanation category III the relationship between the resistance and current quantities leads to the outcome of change of potential difference (relational reasoning). The relationship is Ohms' law, which is not an explanation of the mechanism of the potential difference change, but rather predicts the behavior of the system.

In all of the questions there is a significant percentage of responses that present concepts of electrical theory without a rationale or a logical connection. Moreover, around onequarter of the students did not answer. These results show the high degree of difficulty students encounter when they have to relate the explanatory model of electric current with the concepts of electric field and potential difference at macroscopic and microscopic levels.

The results suggest that when students construct models to explain the role of electric field and potential difference concepts in the working of dc circuits, they can use specific types of reasoning such as simple causal, linear causal, or relational. Further, these results show that the lack of comprehension of the mechanisms underlying the model can lead to different and even opposite conclusions even when using the same model.

A fraction of the students use a simple causal reasoning to explain the relations between the explanatory model of the circuit and the electric field or the potential difference. Very few students, around 5\%-12\%, situate the explanations at the microscopic level (charges or electrons). At the microscopic level, the explanations use a simple causeeffect scheme: the concentration of charges produces the electric field or the potential difference. Almost all answers that mention the concentration of charges situated the charges inside the wire (violation of the principle of conservation of charge) or at the poles of the battery (incomplete explanation). Most of the explanations that use simple causal reasoning are made at the macroscopic level, from $10.5 \%$ to $39 \%$. The reasoning claims that the current is the cause of the electric field. The reasoning describes a physical regularity in a simplistic manner: "the electric current," to arrive at an incorrect conclusion. This type of reasoning has very little explanatory power and fails to describe the mechanism that explains the cause-effect relation.

Some other students presented explanations based on a more complex form of reasoning, namely, linear causal. In question Q1, all explanations that use linear causal reasoning are situated at the macroscopic level. These responses set up a chain of events: the battery produces a potential difference and this produces the electric field. The very few responses that focus on the microscopic level (around 7\% in questions Q2B y Q3B) exhibit a cause-effect chain: the battery produces a concentration of charges and this concentration produces a potential difference. The omission of any explanation of the causal mechanism at both the microscopic and macroscopic levels reveals the lack of complexity in the explanatory reasoning and the diversity of responses grounded in poorly understood concepts.

The reasoning used by the majority of the students in Q2 and Q3 is a relational reasoning at the macroscopic level (25\%-38.5\%), which relates two magnitudes (current and resistance or conductivity of the material) that are included 
in a macroscopic explanatory model based on Ohm's law and the conservation of the current. These explanations explicitly state that the underlying mechanism is the mathematical relation between the variables current and resistance. Further, they constitute a model of reasoning that is more relational than cause effect, as the value of the electric field or of the potential difference depends on a mathematical relation (Ohm's law). This reasoning is an interactive process in time and is auto-adjustable between the variables' current and resistance to obtain a concrete result of the potential difference [29]. Thus, the relational form of reasoning is more complex and has more explanatory power than the two forms of reasoning previously discussed. This result differs from those of previous studies with secondary-level students, which found that the vast majority of the students used a simple causal form of reasoning with little comprehension of the concepts involved $[3,4]$. The results of this study appear to indicate a certain progression in the complexity of the reasoning deployed as one goes higher up the educational levels, which is consistent with the conclusions of the study conducted by Borges and Gilbert [38]. However, students' difficulties to link the model of the circuit with the magnitudes of electric field and potential at the microscopic level still persist, which leads them to conclusions that are scientifically incorrect.

\section{CONCLUSIONS}

This study has allowed us to gain deeper insight into how students make sense of dc circuit situations in which the concepts of electric field, potential difference, and current may all appear to be pertinent. After standard instruction, a small fraction of students provided error-free answers that do not focus on the relations between charge distribution, electric field, and potential difference. The vast majority of the students propose an explanatory model in one of three ways:

(i) A relational form of reasoning that sets up a relation between current and resistance producing the electric field and/or potential difference;

(ii) A linear causal reasoning that regards the battery as the cause of the potential difference and, this in turn, the cause of the electric field. This same line of reasoning is followed at the microscopic level, replacing the battery with a charge density distribution in the wire of the circuit;

(iii) A simple causal line of reasoning that states that the current or the variation in charge concentrations produces the electric field and/or the potential difference. Moreover, the vast majority of the students use explanations at macroscopic level, although they study and apply to circuits Ohm's law at the microscopic level $(J=\sigma E$, where $J$ is the current density and $\sigma$ is the conductivity) during the teaching of the electric circuit chapter. It seems that students have more difficulty describing the model of current at the microscopic level than at the macroscopic level. This might be because most traditional teaching focuses on solving problems with quantitative relationships at the macroscopic level.

The difficulties encountered could be due, as some studies have suggested $[15,23,24]$, to a failure to bring the syllabus up to date by focusing teaching on dc circuits, not just on the relations between the magnitudes of the circuit at the macroscopic level (Kirchhof's laws) but also looking into the explanatory mechanisms of the production of current at the microscopic level. The presentation, for instance, of the potential difference in a circuit as the battery's capacity to do work, as energy per unit of charge, involves an orientation that is in part mathematical [13]. This traditional approach sidesteps the relation between potential difference and the charge distribution throughout the circuit. This may influence students to attempt to arrive at their own tentative explanations inductively to establish the macro-micro relations in the circuit [39].

In the last decade, Chabay and Sherwood have proposed, for electricity introductory courses at university, a change in the syllabus that emphasizes the crucial role played by charges on the surfaces of the elements of the circuit, which makes it possible to describe circuit behavior directly in terms of charge and electric field. This approach is a more fundamental description of the circuits that allows one to unify the treatment of electrostatics and circuits $[15,26]$. However, the new curricula proposals based on a gradient surface charge microscopic model require an elaborate mechanism underlying relational and multilevel reasoning. Our study shows aspects, little mentioned in education research at the university level, of the types of reasoning used by students when establishing macro-micro relations and possible difficulties with the multilevel reasoning processes. We find that the types of reasoning deployed by students are of low explanatory power; even those students who use a relational form of reasoning only apply it to the macroscopic level and incorrectly induce similar consequences for the microscopic level. This lack of resources for students to develop complex reasoning may constitute a major obstacle in teaching high-demand models of reasoning, such as the gradient surface charge microscopic model. Such models represent micro-macro relations as a multilevel form of reasoning. It will, therefore, be necessary not only to change the curriculum, but also work with students explicitly on ways of scientific reasoning leading to macro-micro relations in a dc circuit. Not only explanatory mechanisms at the level of atomic dynamics should be presented to students, but also the opportunity to apply complex forms of reasoning that can stimulate discussion. We therefore suspect that this could be one of the reasons why reforms of the course content curricula of introductory electrical circuits do not progress 
as rapidly as research results would lead one to expect. There are few studies on evaluating the improving students' understanding of electric circuits following the "matter and interaction" approach [26]; Kohlmyer et al.'s work indicates that the higher performance obtained by students who follow this approach on the BEMA postquestionnaire measures only the total effect of content and pedagogy [[40], p. 020105-9]. In the future it will be necessary to analyze the individual elements of teaching methodology and the difficulties of reasoning of students.

The findings of this study will guide the next stage of our work that involves designing, implementing, and evaluating a teaching-learning sequence (TLS) in classroom environments for different countries in introductory university physics courses. The study will focus on a stepby-step design strategy that gradually provides an interpretative model of electrical circuits based on concepts and conceptual changes. In particular, the microscopic model of electric current will be introduced from the beginning to provide continuity with the studied theory of electrostatics (electric charge, field, and potential) and, conceptual elements (gradient of surface-charge distribution, electric field in the transient, and steady states of current) will be gradually introduced. The TLS design takes into account not only the conceptual updating of the interpretative model, but also the learning demands that require the teaching objectives from the point of view of both conceptual and reasoning complexity [41].

\section{ACKNOWLEDGMENTS}

We thank the Spanish Government MINECO/FEDER Project EDU2015-65359-P for financial support. We would like to thank Dr. Jaume Ametller for his critical reading and his suggestions.

\section{APPENDIX}

Q1. In the chapter about electric fields, we studied how in the interior of a charged cylindrical conductor in electrostatic equilibrium there is no electric charge (all of the charge is on the surface of the conductor) and hence the electric field inside is zero. However, in the chapter on dc electrical circuits we studied that there is a uniform electric field inside the cylindrical conductor cable when a current is circulating with constant amperage.

Why does this electric field appear inside the cable? Explain your answer.

Q2. In the expanded circuit shown in Fig. 1, a short carbon resistor is connected to a battery by copper wire. The circuit has been connected for a long time, and there is a constant current flowing through the circuit. Copper and carbon are both conductors, but carbon has much lower conductivity than copper.

Q2A.Choose the diagram below which best represents the electric field at points $A$ and $B$.

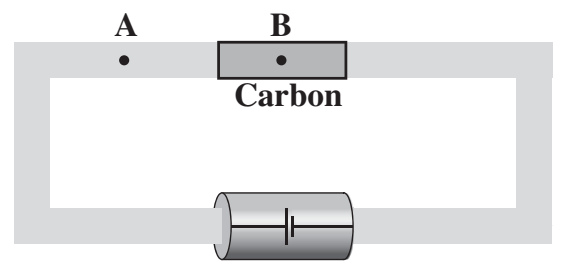

FIG. 1. A simple circuit with a carbon resistor.

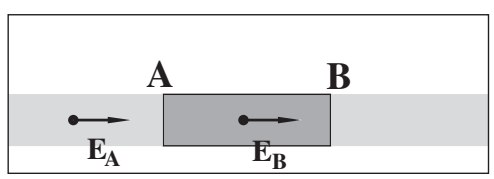

FIG. 2. The electric field at a point outside the resistor is equal to the electric field at a point inside the resistor.

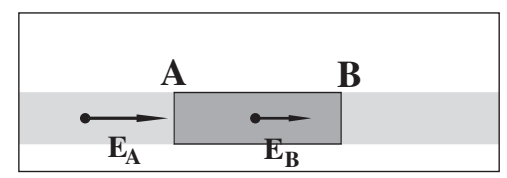

FIG. 3. The electric field at a point outside the resistor is greater than the electric field at a point inside the resistor.

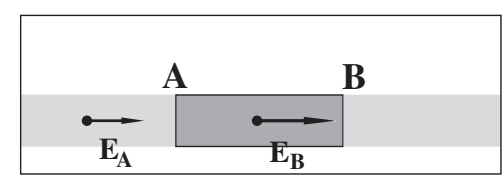

FIG. 4. The electric field at a point outside the resistor is less than the electric field at a point inside the resistor.

(1) $E_{A}$ is equal to $E_{B}$ (see Fig. 2).

(2) $E_{A}$ is greater than $E_{B}$ (see Fig. 3).

(3) $E_{A}$ is less than $E_{B}$ (see Fig. 4).

(4) A different set of electric field vectors (please describe):

Explain the reasoning you used to answer the previous question.

Q2B. Is the potential difference between points $A$ and $B$ related to the charge distribution? Explain why or why not.

Q3. A circuit consists of a battery connected to a copper wire (see Fig. 5). The wire is narrowed in a part of the circuit, as shown below. The circuit has been connected for

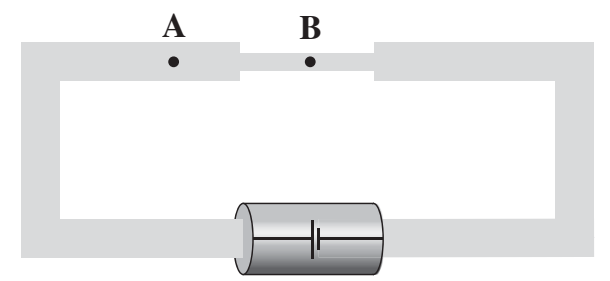

FIG. 5. A simple circuit with a "resistor" (a thin section of the wire). 


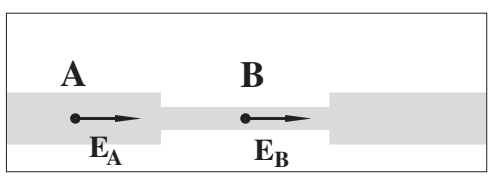

FIG. 6. The electric field at a point outside the thin section is equal to the electric field at a point inside the thin section.

a long time, and there is a constant current flowing through the circuit.

Q3A. Choose the diagram below which best represents the electric field at points $A$ and $B$.

(1) $E_{A}$ is equal to $E_{B}$ (see Fig. 6).

(2) $E_{A}$ is greater than $E_{B}$ (see Fig. 7).

(3) $E_{A}$ is less than $E_{B}$ (see Fig. 8).

(4) A different set of electric field vectors (please describe):

Explain the reasoning you used to answer the previous question.

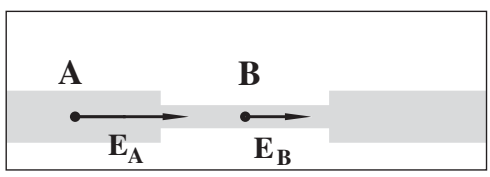

FIG. 7. The electric field at a point outside the thin section is greater than the electric field at a point inside the thin section.

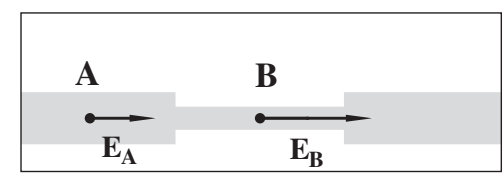

FIG. 8. The electric field at a point outside the thin section is less than the electric field at a point inside the thin section.

Q3B.- Is there a potential difference between points $A$ and $B$ ? Explain why or why not.
[1] N. Fredette and J. Lochhead, Student conceptions of simple circuits, Phys. Teach. 18, 194 (1980).

[2] C. von Rhöneck, Student conceptions of the electric circuit before physics instruction, in Proceedings of the International Workshop on Problems Concerning Students' Representation of Physics, and Chemistry Knowledge, edited by W. Jung, H. Pfundt, and C. von Rhoneck (Pädagogische Hochschule, Ludwigsburg, 1981), pp. 194-213.

[3] R. Millar and T. King, Students' understanding of voltage in simple series electric circuits, Int. J. Sci. Educ. 15, 339 (1993).

[4] A. Barbas and D. Psillos, Causal Reasoning as a base for advancing a systemic approach to simple electrical circuits, Res. Sci. Educ. 27, 445 (1997).

[5] B. Eylon and U. Ganiel, Macro-micro relationship: the missing link between electrostatics and electrodynamics in students' reasoning, Int. J. Sci. Educ. 12, 79 (1990).

[6] E. Bagno and B.S. Eylon, From problem solving to a knowledge structure: An example from the domain of electromagnetism, Am. J. Phys. 65, 726 (1997).

[7] R. L. C. Hazelton, M. R. Stetzer, P. R. L. Heron, and P. S. Shaffer, Investigating student ability to apply basic electrostatics concepts to conductors, AIP Conf. Proc. 1513, 166 (2013).

[8] A. Bensheguir and J.L. Closset, The electrostaticselectrokinetics transition: historical and educational difficulties, Int. J. Sci. Educ. 18, 179 (1996).

[9] C. Guruswamy, M. D. Somers, and R. G. Hussey, Students' understanding of the transfer of charge between conductors, Phys. Educ. 32, 91 (1997).

[10] See, for example, R. Duit, W. Jung, and C. von Rhöneck, Aspects of Understanding Electricity, in
Proceedings of an International Workshop, edited by V. Schmidt and K. Kiel (Ludwigsburg, Germany, 1984); M. Shipstone, C. von Rhöneck, W. Jung, C. Kärrqvist, J. J. Dupin, S. Johsua, and P. Licht, A study of students' understanding of electricity in five European countries, Int. J. Sci. Educ. 10, 303 (1988); J. Guisasola, Teaching and learning electricity: The relations between macroscopic level observations and microscopic level theories, in International Handbook of Research in History, Philosophy and Science Teaching, edited by M. R. Matthews (Springer, New York, 2014), pp. 129-156.

[11] J. L. Closset, Sequential reasoning in electricity, in Research on Physics Education: Proceedings of the First International Workshop edited by G. Delacote, A. Tiberghien, and J. Schwartz (Editions du CNRS, Paris, France, 1983), pp. 313-319.

[12] J. Guisasola, J. L. Zubimendi, and K. Zuza, How much have students learned? Research based teaching on electrical capacitance, Phys. Rev. ST Phys. Educ. Res. 6, 020102 (2010).

[13] H. Härtel, The electric voltage: What do students understand? What can be done to help for a better understanding? in Aspects of Understanding Electricity: Proceedings of an International Workshop edited by R. Duit, W. Jung, and C. von Rhöneck (IPN-Kiel, Ludwigsburg 1985), pp. 353-362.

[14] B. A. Thacker, U. Ganiel, and D. Boys, Macroscopic phenomena and microscopic processes: Student understanding of transients in direct current electric circuits, Am. J. Phys. 67, S25 (1999).

[15] R. W. Chabay and B. A. Sherwood, Restructuring the introductory electricity and magnetism course, Am. J. Phys. 74, 329 (2006). 
[16] S. M. Stocklmayer and D. F. Treagust, A historical analysis of electric current in textbooks: a century of influence on physics education, Sci. \& Educ. 3, 131 (1994).

[17] P. A. Tipler and G. Mosca, Physics for Scientists and Engineers, 5th ed. (W. H. Freeman and Company, New York, 2004).

[18] P. M. Fishbane, S. Gasiorowics, and S. T. Thornton, Physics for Scientists and Engineers, 2nd ed. (Prentice Hall Upper Saddle River, New Jersey, 1996).

[19] S. M. Stocklmayer and D. F. Treagust, Images of electricity: How novices and experts model electric current?, Int. J. Sci. Educ. 18, 163 (1996).

[20] P. E. Hirvonen, Surface-charge-based micro-models- a solid foundation for learning about direct current circuits, Eur. J. Phys. 28, 581 (2007).

[21] S. M. Stocklmayer, Teaching direct current theory using a field model, Int. J. Sci. Educ. 32, 1801 (2010).

[22] A. Sommerfeld, Electrodynamics (Academic, New York, 1952).

[23] O. D. Jefimenko, Electricity and Magnetism (AppletonCentury-Crofts, New York, 1966).

[24] H. Härtel, The electric circuit as a system: A new approach, Eur. J. Sci. Educ. 4, 45 (1982).

[25] R. D. Knight, Physics a Strategic Approach, 3rd ed. (Pearson, Boston, 2013) Chap. 30.

[26] R. W. Chabay and B. A. Sherwood, Matter and Interactions, 4th ed. (Wiley, New Jersey, 2015), Chap. 18.

[27] D. J. Griffith, Introduction to Electrodynamics, 4th ed. (Pearson Education Ltd., Harlow, 2014).

[28] N. J. Nersessian, Mental modeling in conceptual change, in International Handbook of Research on Conceptual Change, edited by S. Vosniadou (Routledge, London, 2008), pp. 391-416.

[29] M. Windschitl, J. Thompson, and M. Braaten, Beyond the scientific method: Model-based inquiry as a new paradigm of preference for school science investigations, Sci. Educ. 92, 941 (2008).

[30] F. Halbwachs, Causalité linéaire et causalité circulaire en physique (linear causality, and circular causality in physics) Les theories de la causalité, edited by M. Bunge,
F. Halbwachs, T. S. Khun, J. Piaget, and L. Rosenfeld (Presses Universitaires de France, Paris, 1971), pp. 39-111.

[31] T. A. Grotzer, Learning to understand the forms of causality implicit in scientific explanations, Stud. Sci. Educ. 39, 1 (2003).

[32] D. N. Perkins and T. A. Grotzer, Dimensions of causal understanding: The role of complex causal models in students' understanding of science, Stud. Sci. Educ. 41, 117 (2005).

[33] T. A. Grotzer and M.S. Tutwiler, Simplifying causal complexity: How interactions between modes of causal induction and information availability lead to heuristicdriven reasoning, Mind, Brain, and Educ. 8, 97 (2014).

[34] L. Cohen, L. Manion, and K. Morrison, Research Methods in Education (Routledge Taylor and Francis Group, London, 2007).

[35] M. Cortazzi, Narrative Analysis (Palmer Press, London, 1993).

[36] H. Härtel, Constant electric current, and the distribution of surface charges available at: http://www.astrophysik .uni-kiel.de/ hhaertel/PUB/voltage_IRL.pdf (2005). This is a revised part of a full paper, published under the title "A Qualitative Approach to Electricity" at the Institute for Research on Learning, Report No. IRL87-000, (1987).

[37] R. N. Giere, Using models to represent reality, in ModelBased Reasoning in Scientific Discovery, edited by L. Magnani, N. J. Nersessian, and P. Thagard (Kluwer and Plenum Publishers, New York, 1999), pp. 41-57.

[38] A. T. Borges and J. K. Gilbert, Mental models of electricity, Int. J. Sci. Educ. 21, 95 (1999).

[39] A. A. DiSessa, Toward an epistemology of physics, Cognit. Instr. 10, 105 (1993).

[40] M. A. Kohlmyer, M. D. Caballero, R. Catrambone, R. W. Chabay, L. Ding, M. P. Haugan, M. J. Marr, B. A. Sherwood, and M.F. Schatz, Tale of two curricula: The performance of 2,000 students in introductory electromagnetism, Phys. Rev. ST Phys. Educ. Res. 5, 020105 (2009).

[41] J. Leach and P. Scott, Designing and evaluating science teaching sequences: An approach drawing upon the concept of learning demand and a social constructivist perspective on learning, Stud. Sci. Educ. 38, 115 (2002). 